\title{
Proceso enseñanza- aprendizaje constructivista en la asignatura de Química general QQ-103 de la UNAH (año 2016)
}

\author{
María de los Angeles Ramírez ${ }^{1}$ \\ Karen Orellana ${ }^{2}$ \\ Fredy Rodriguez Rivas ${ }^{3}$
}

\section{RESUMEN}

El objetivo del estudio consistió en analizar e implementar estrategias didácticas coherentes al enfoque de competencias y aprendizaje efectivo/significativo para la comprensión y resolución de problemas relacionados a las ciencias químicas, estas estrategias comprendieron: Mapas Conceptuales, diagrama de flujo, UVE de Gowin, resolución de problemas y estudio de casos aplicados al tema de medición de masa, volumen y densidad de sustancias sólidas y líquidas de la materia, mediante una guía didáctica diseñada en función de los contenidos analizados.

Para el estudio se utilizó una muestra aleatoria de 55 estudiantes de tres secciones de Química General QQ103 durante el primer período académico del año 2016, una de ellas se estableció como grupo control conformado por 15 estudiantes y las otras dos secciones con un total de 40 estudiantes. Se diagnosticó el aprendizaje efectivo/significativo mediante un pre test, considerando sus actitudes positivas y negativas hacia el conocimiento de la química, así como su comprensión del temario antes y después de la aplicación de las estrategias didácticas implementadas, asimismo como de una guía didáctica validada. Se efectuó un pos test para indagar el logro de competencias básicas. La información obtenida se procesó empleando el programa estadístico para las ciencias sociales (SPSS). Los resultados fueron expresados en términos numéricos y porcentuales mostrado una actitud positiva muy favorable en los grupos control y experimental, ambos con conocimientos y competencias previas similares. El grupo experimental mostró mayor fijación de conocimiento y un aumento en el desarrollo de competencias básicas.

Palabras clave: Aprendizaje, competencias, estrategias didácticas

\footnotetext{
${ }^{1}$ Cursante de la Maestría en Docencia en Ciencias, Universidad Nacional Autónoma de Honduras

${ }^{2}$ Master Interuniversitario en Descubrimiento de Fármacos, Universidad Complutense de Madrid, España.

3 Pasante del programa de Doctorado en Química Fina, Universidad de Córdoba, España: frelex22@hotmail.com
} 


\section{ABSTRACT}

The objective of the study was to analyze the implementation of the methodology based on competences and effective / significant learning, using innovative didactic strategies for understanding and solving problems related to the chemical sciences, these strategies included: conceptual Maps, flow chart, UVE of Gowin, problem solving and case studies applied to the subject of mass, volume and density measurement of solid and liquid substances of the subject, through a didactic guide designed according to the contents analyzed. For the study a random sample of 55 students was used, belonging three sections of General Chemistry QQ103 of the first academic period 2016, one of them was established as a control group consisting of 15 students and the other two sections with a total of 40 students. Effective / significant learning was diagnosed by means of a pre-test, considering their positive and negative attitudes towards the knowledge of chemistry, as their comprehension of the syllabus before and after the application of the didactic strategies implemented, as well as a didactic guide validated pos test was made to investigate the achievement of generic competences. The information obtained was processed using the statistical program for the Social Sciences (SPSS), and the results were expressed in numerical and percentage terms.

Keywords: Learning, competences, teaching strategies 


\section{INTRODUCCIÓN}

La época actual caracterizada por el desarrollo de las nuevas tecnologías, cambios producidos en los procesos económicos y financieros, aparición de nuevos problemas sociales y culturales, y la necesidad de hacer eficientes los recursos que se disponen para obtener los mejores resultados en cualquier situación que se presente, obligan a desarrollar nuevos paradigmas educativos que nos ayuden a entender la realidad que nos rodea. (Mayer, 2004), (Angulo, 2005).

Para responder a un mundo globalizado del siglo XXI de una manera adecuada, eficaz y eficiente el nuevo paradigma educativo basado en competencias ayuda a promover un cambio imprescindible sobre el desarrollo de las habilidades para construir competencias en el estudiante, es decir ubicar, recuperar, transformar y relacionar los conocimientos que ya se poseen para lograr un desempeño adecuado (Ortiz Uribe, 2010).

Por estas razones la educación en el presente y el futuro inmediato requiere redoblar sus esfuerzos en promover desarrollo de habilidades que sustentan los principios que propone la UNESCO para el sustento de la educación del presente siglo, los cuales son los llamados pilares de la educación: aprender a ser, aprender a pensar, aprender a conocer, aprender a hacer y aprender a convivir; los cuales involucran aprender a aprender (Delor, 1994).

El enfoque basado en competencias actualmente es un referente para el diseño curricular y didáctico a nivel universitario para promover en el estudiante las competencias de gestión del conocimiento y el autoaprendizaje, entre otros. En este modelo los alumnos son vistos como trabajadores del conocimiento, es decir como individuos equipados con habilidades, destrezas, actitudes y valores que les permite adaptarse al continuo cambio que se da en la sociedad del conocimiento (Montenegro, 2003). Por tal razón se debe tener claro de que una competencia es una combinación de atributos en relación a conocimientos, habilidades, actitudes y responsabilidades que describen los resultados del aprendizaje de un programa educativo o a lo que los alumnos son capaces de demostrar al final del proceso educativo (González \& Wagenaar , 2003)

La Universidad Nacional Autónoma de Honduras (UNAH) formadora del talento humano del país, debe fortalecer su capacidad de generación y gestión de conocimiento en bien de la mejora de la calidad de los procesos formativos y lograr una 
innovación educativa que se dirija a la formación de personas íntegras y capaces de desarrollar competencias básicas tanto para el bienestar personal como profesional. Este enfoque basado en competencias propone una serie de condicionantes en la planificación y desarrollo del proceso formativo: El estudiante debe ser activo y autónomo en la construcción de su propio conocimiento; la enseñanza debe fomentar aprendizajes no solo conceptuales sino también procedimentales y actitudinales; se deben desarrollar las competencias necesarias que le permitan a los estudiantes desenvolverse creativa y adecuadamente en un entorno, promoviendo situaciones significativas en condiciones "similares" al contexto real, es decir colaboración, experimentación, resolución de conflictos y toma de decisiones, entre otros (Monereo y Pozo, 2003), (Diaz barriga Arcejo, 2010).

En las últimas décadas se han venido realizando numerosas investigaciones sobre los problemas específicos de la enseñanza y del aprendizaje de las ciencias y se ha revisado sus bases epistemológicas, lo que ha conducido a la puesta en práctica en el aula de nuevos modelos de enseñanza de las ciencias. Todo esto ha venido generando modelos dominantes en cada etapa histórica de la humanidad, el tradicional de transmisión-recepción, el del descubrimiento y el actual constructivismo. Todos ellos han ido aportando una nueva visión del proceso enseñanza-aprendizaje (Maldonado, 2001).

Con el presente trabajo se pretende exponer los resultados de una experiencia enseñanza-aprendizaje con los estudiantes de Química General QQ-103 de la UNAH, en la cual se integran la inclusión y el desarrollo de competencias por medio del proceso de enseñanza aprendizaje Constructivista.

\section{METODOLOGÍA}

El diseño del estudio es experimental de corte transversal, es decir en un solo momento, haciendo uso de la observación y recolección de datos por medio de un cuestionario, el cual responde únicamente al comportamiento de los estudiantes de Química General QQ103 en la UNAH, área de servicio para estudiantes del área de la salud: Enfermería, microbiología, odontología, biología, entre otras. El estudio presenta un enfoque de tipo cuantitativo, ya que este usa la recolección de datos para comprobar hipótesis con base en la medición numérica y el análisis estadístico para establecer patrones de comportamiento y probar teorías (Sampieri, Metodología de la Investigación, 2014). 
La población objeto de estudio fue conformada por estudiantes de la Universidad Nacional Autónoma de Honduras matriculados en la asignatura de Química General QQ 103 durante el primer período académico del año 2016 (con 4 secciones inscritas en la oficina de registro, cada una con un total de quince a veintidós estudiantes matriculados). Tres de ellas fueron seleccionadas como objeto de estudio en esta investigación analizando un total de cincuenta y cinco estudiantes. De ellos, quince constituyeron el grupo control y cuarenta el grupo experimental (veintidós estudiantes de la sección 1000 y 18 alumnos de la sección 1100).

En los dos grupos (control y experimental) se aplicó un pre test y un post test comparando los resultados obtenidos en ambos. En los cuales se evaluaron actitudes hacia el estudio de las ciencias y conocimientos sobre la temática a desarrollar. Además se evaluó el logro de competencias a través de las estrategias constructivistas en un grupo experimental y tradicional en el grupo control.

El pre test incluyó cincuenta (50) preguntas para evaluar actitudes y conocimientos de la temática en ciencia a desarrollar en la unidad didáctica y 15 preguntas de la temática de densidad para identificar conocimiento previo. De la pregunta número 1 a la 32 del pre test se evaluó la actitud del estudiante con respecto al estudio de las ciencias y de la pregunta 33 a la 50 se evaluaron competencias básicas hacia la ciencia.

Seguidamente se aplicaron las estrategias didácticas de aprendizaje al grupo experimental, es decir la etapa de intervención en el estudio. Posteriormente se realizó un pos test orientado a evaluar las mismas características con los objetos de estudios anteriormente expuestos. Consecutivamente, se procedió a realizar la aplicación de estrategias didácticas constructivistas al grupo experimental, las cuales consistieron en una serie de técnicas didácticas de confirmación de competencias básicas en las que se pueden mencionar mapas conceptuales, UVE de Gowin, estudios de casos y resolución de problemas. Esto con el objetivo de corroborar la interpretación del tema de estudio desarrollado en esta investigación. Por su parte con el grupo control se empleó estrategia didácticas tradicionales como son las clases magistrales y resolución de ejercicios prácticos. Al igual que al grupo experimental se les aplicó el mismo pos test. De esta manera se realizó la comparación entre ambos grupos corroborando los objetivos propuestos en esta investigación.

La información obtenida en los cuestionarios y escalas de observación se procesó en el programa estadístico SPSS versión 20. Para llevar a cabo el procesamiento de los mismos, se empleó la distribución de frecuencias (ordenamiento en forma de tabla de 
los datos estadísticos asignando a cada dato su frecuencia correspondiente), comparación de medias con el fin de obtener el conteo del número de repuestas asociadas con los valores de cada variable, cuyos resultados fueron expresados en términos numéricos y porcentuales.

\section{RESULTADOS}

1. Los resultados obtenidos acerca de la actitud positiva hacia los conocimientos de las ciencias químicas en los grupos control y experimental tanto en el pre test como el pos test se muestran en los gráficos 1 y 2.

Gráfico 1. Actitud de los grupos control y experimental hacia las ciencias químicas (pre-test)

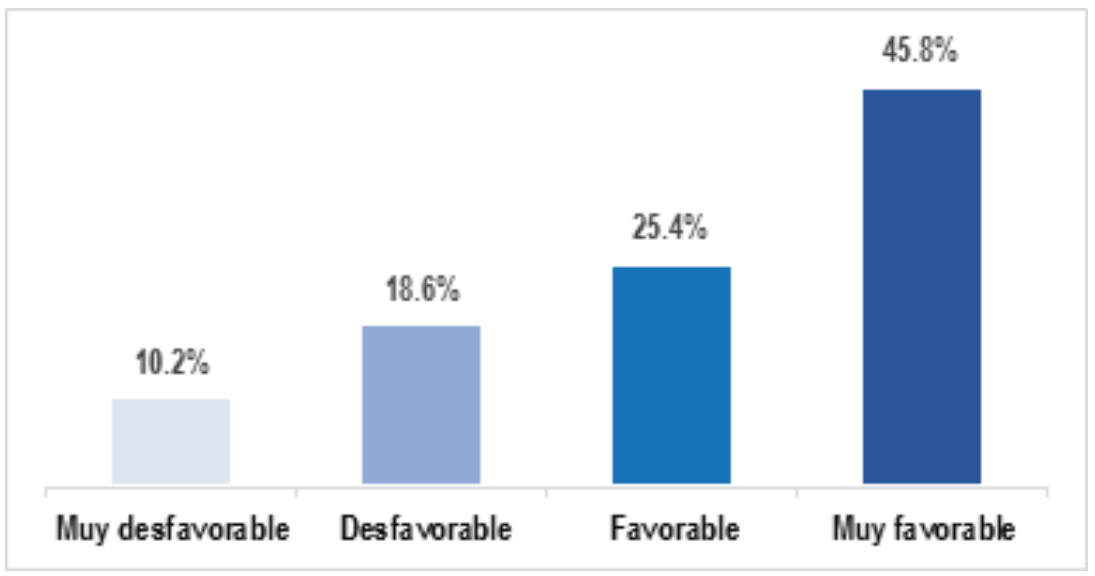

Fuente: Elaboración propia

2. Los resultados obtenidos en la evaluación del conocimiento sobre el tema de ciencia a desarrollar en los grupos control y experimental en el pre test se muestran en las tablas 1 de comparación de respuestas correctas, y en las tablas 2 y 3 de prueba de Hipótesis; teniendo como base las siguientes especulaciones: 
Gráfico 2. Actitud de los grupos control y experimental hacia las ciencias químicas post-test

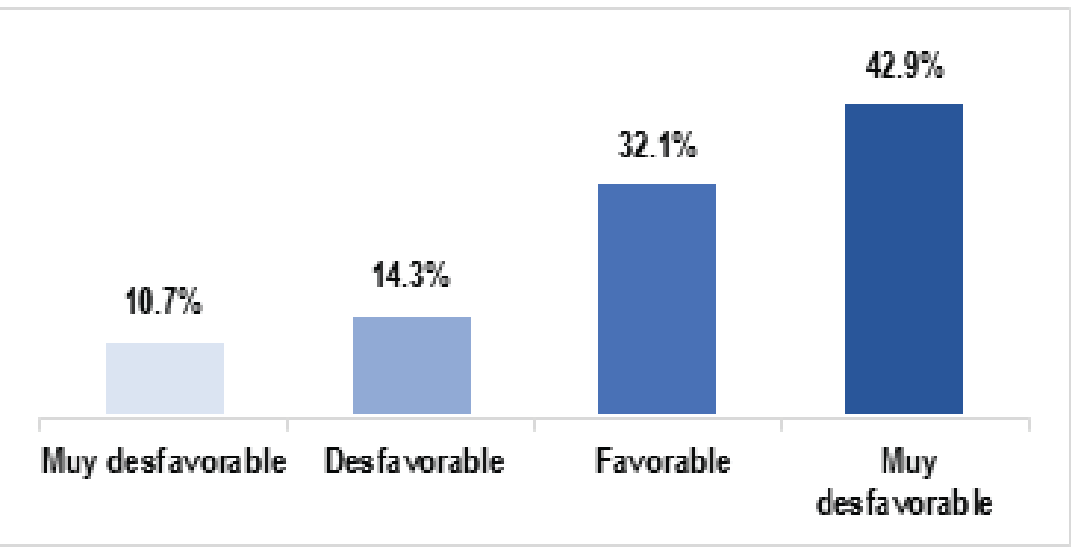

Fuente: Elaboración propia

Pre-test en conocimiento comparando el grupo experimental con el grupo tradicional $\mathrm{H} 0=$ Se logra un igual conocimiento mediante la aplicación de estrategias didácticas constructivistas y estrategias didácticas tradicionales.

H1= Se logra un mayor conocimiento mediante la aplicación de estrategias didácticas constructivistas que al utilizar estrategias didácticas tradicionales.

Tabla 1. Comparación de las medias de respuesta correctas entre los grupos experimental y tradicional pre-test

\begin{tabular}{|c|c|c|c|c|c|}
\hline \multicolumn{7}{|c|}{ Estadísticos de muestras relacionadas } \\
\hline \multicolumn{2}{|c|}{} & Media & $\mathrm{N}$ & $\begin{array}{c}\text { Desviación } \\
\text { típica }\end{array}$ & $\begin{array}{c}\text { Error típico } \\
\text { de la media }\end{array}$ \\
\hline \multirow{2}{*}{$\begin{array}{c}\text { Par } \\
1\end{array}$} & Modelo Experimental & 8.27 & 15 & 1.534 & 0.396 \\
\cline { 2 - 6 } & Método Tradicional & 7.53 & 15 & 2.031 & 0.524 \\
\hline
\end{tabular}

Fuente: Elaboración propia 
Tabla 2. Prueba de hipótesis entre el conocimiento del grupo experimental y tradicional en el pre-test

\begin{tabular}{|c|c|c|c|c|c|}
\hline \multicolumn{2}{|c|}{} & \multicolumn{4}{c|}{ Prueba de muestras relacionadas } \\
\cline { 2 - 6 } & Media & $\begin{array}{c}\text { Desviación } \\
\text { típica }\end{array}$ & $\begin{array}{c}\text { Error } \\
\text { típico de } \\
\text { la media }\end{array}$ & $\begin{array}{c}95 \% \text { Intervalo de } \\
\text { confianza para la } \\
\text { diferencia Inferior }\end{array}$ \\
\hline \begin{tabular}{c}
$|c|$ \\
\multicolumn{2}{|c|}{ Par } \\
1
\end{tabular} & $\begin{array}{c}\text { Modelo Experimental - } \\
\text { Método Tradicional }\end{array}$ & .733 & 2.492 & .643 & -.647 \\
\hline
\end{tabular}

Fuente: Elaboración propia

Tabla 3. Prueba de hipótesis entre el conocimiento del grupo experimental y tradicional en el pre-test

\begin{tabular}{|c|c|c|c|c|c|}
\hline \multicolumn{6}{|c|}{ Prueba de muestras relacionadas } \\
\hline & & Diferencias relacionadas & \multirow[b]{2}{*}{$t$} & \multirow[b]{2}{*}{ gl } & \multirow[b]{2}{*}{$\begin{array}{c}\text { Sig. } \\
\text { (bilateral) }\end{array}$} \\
\hline & & $\begin{array}{l}\text { 95\% Intervalo de } \\
\text { confianza para la } \\
\text { diferencia Superior }\end{array}$ & & & \\
\hline $\begin{array}{c}\text { Par } \\
1\end{array}$ & $\begin{array}{l}\text { Modelo Experimental - } \\
\text { Método Tradicional }\end{array}$ & 2.113 & 1.140 & 14 & 0.274 \\
\hline
\end{tabular}

Fuente: Elaboración propia

3. Los resultados obtenidos en la evaluación de los conocimientos sobre el tema de ciencia a desarrollar en los grupos control y experimental en el post test se muestran en la tabla 4 de comparación de respuestas correctas, y tabla 5 y 6 de prueba de Hipótesis. Parte de las siguientes presunciones:

Post-test en conocimiento Comparando el grupo Experimental con el grupo tradicional

$\mathrm{H} 0=$ Se logra un igual conocimiento mediante la aplicación de estrategias didácticas constructivistas y estrategias didácticas tradicionales.

H1= Se logra un mayor conocimiento mediante la aplicación de estrategias didácticas constructivistas que al utilizar estrategias didácticas tradicionales.

4. Resultados obtenidos en la evaluación de logro de competencias básicas de los estudiantes de química en los grupos control y experimental en el pre y post test se muestran en los gráficos $3,4,5$ y 6 
Tabla 4. Comparación de la media de respuestas correctas entre el grupo control y experimental en el post-test

\begin{tabular}{|c|c|c|c|c|c|}
\hline \multicolumn{7}{|c|}{ Estadísticos de muestras relacionadas } \\
\hline \multirow{2}{*}{} & Media & $\mathbf{N}$ & $\begin{array}{c}\text { Desviación } \\
\text { típica }\end{array}$ & $\begin{array}{c}\text { Error típico } \\
\text { de la media }\end{array}$ \\
\hline \multirow{2}{*}{$\begin{array}{c}\text { Par } \\
1\end{array}$} & $\begin{array}{c}\text { Modelo } \\
\text { Experimental }\end{array}$ & 9.19 & 16 & 2.228 & 0.557 \\
\cline { 2 - 6 } & Método Tradicional & 7.13 & 16 & 2.705 & 0.676 \\
\hline
\end{tabular}

Fuente: Elaboración propia

Tabla 5. Prueba de hipótesis entre el conocimiento del grupo experimental y tradicional en el post-test

\begin{tabular}{|c|c|c|c|c|c|}
\hline \multicolumn{2}{|c|}{ Prueba de muestras relacionadas } \\
\cline { 3 - 6 } & Media & $\begin{array}{c}\text { Diferencias relacionadas } \\
\text { Desviación } \\
\text { típica }\end{array}$ & $\begin{array}{c}\text { Error } \\
\text { típico de } \\
\text { la media }\end{array}$ & $\begin{array}{c}95 \% \text { Intervalo } \\
\text { de confianza } \\
\text { para la } \\
\text { diferencia } \\
\text { Inferior }\end{array}$ \\
\hline Par1 & $\begin{array}{c}\text { Modelo } \\
\text { Experimental - } \\
\text { Método Tradicional }\end{array}$ & 2.063 & 4.266 & 1.066 & -0.211 \\
\hline
\end{tabular}

Fuente: Elaboración propia

Tabla 6. Prueba de hipótesis entre el conocimiento del grupo experimental y tradicional en el pre-test

\begin{tabular}{|c|c|c|c|c|c|}
\hline \multicolumn{6}{|c|}{ Prueba de muestras relacionadas } \\
\hline & & $\begin{array}{c}\text { Diferencias } \\
\text { relacionadas }\end{array}$ & & & \\
\hline & & $\begin{array}{c}95 \% \text { Intervalo de } \\
\text { confianza para } \\
\text { la diferencia } \\
\text { Superior }\end{array}$ & $t$ & gl & $\begin{array}{c}\text { Sig. } \\
\text { (bilateral) }\end{array}$ \\
\hline $\begin{array}{c}\text { Par } \\
1\end{array}$ & $\begin{array}{c}\text { Modelo Experimental - Método } \\
\text { Tradicional }\end{array}$ & 4.336 & 1.934 & 15 & 0.072 \\
\hline
\end{tabular}

Fuente: Elaboración propia 
Análisis pre-test de competencias básicas en el grupo tradicional y experimental

Gráfico 3. Competencias básicas Pre-test en el grupo control

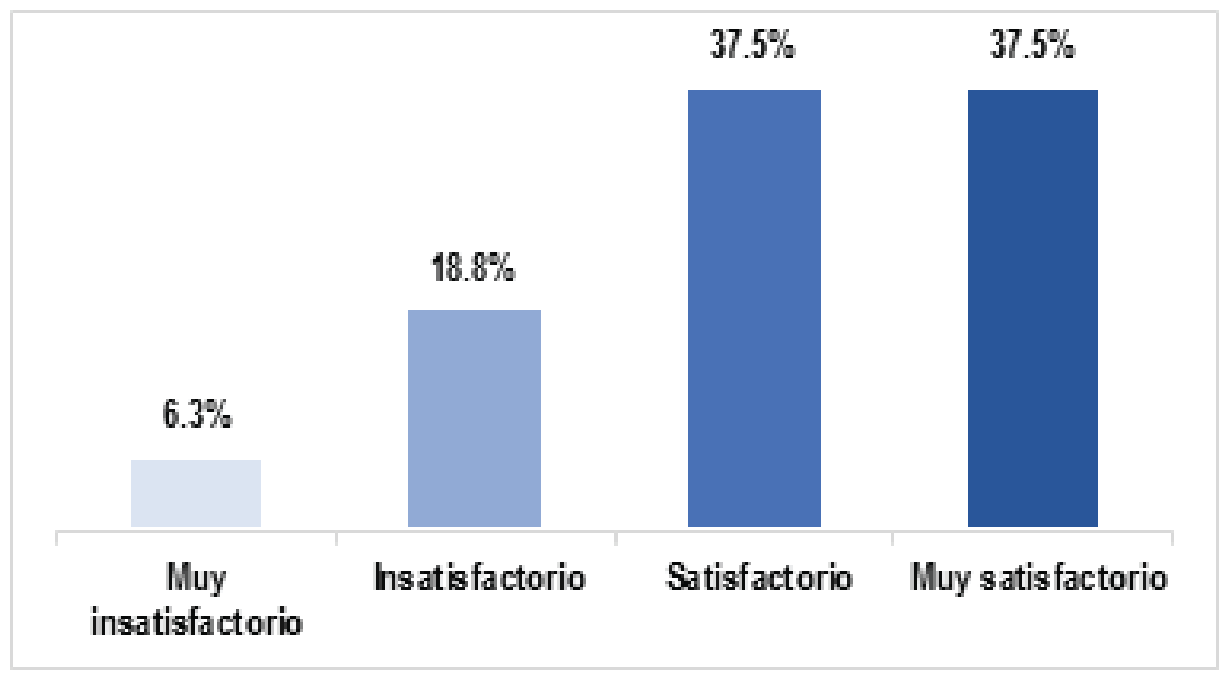

Fuente: Elaboración propia

Gráfico 4. Competencias básicas Pre-test en el grupo Experimental

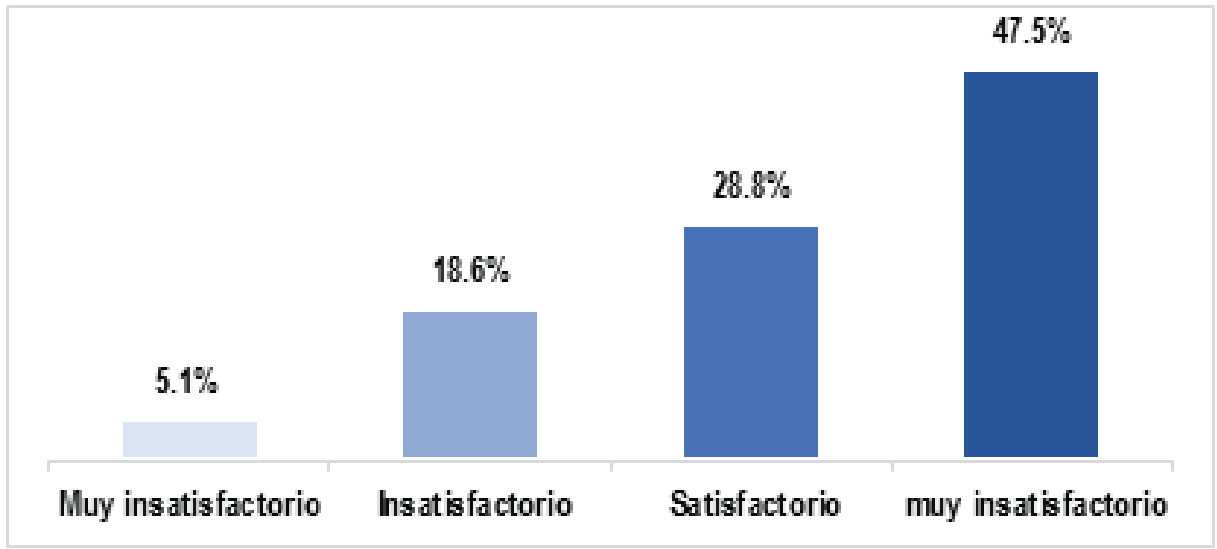

Fuente: Elaboración propia 
Análisis post-test de competencias básicas en el grupo control y experimental

Gráfico 5. Competencias básicas Post-test en el grupo control

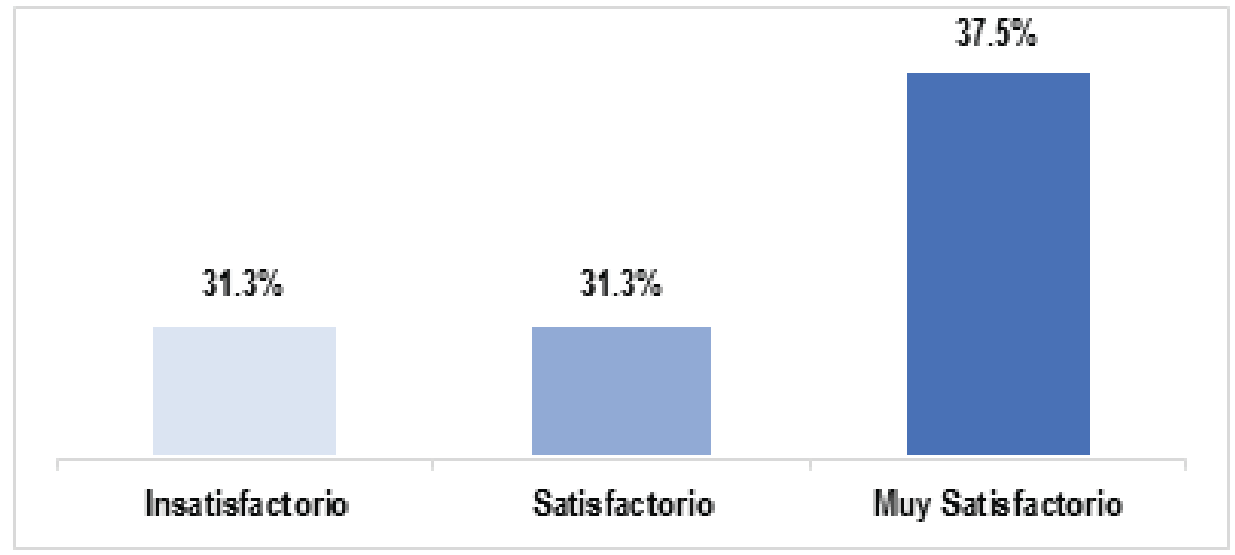

Fuente: Elaboración propia

\section{Gráfico 6. Competencias básicas Post-test en el grupo experimental}

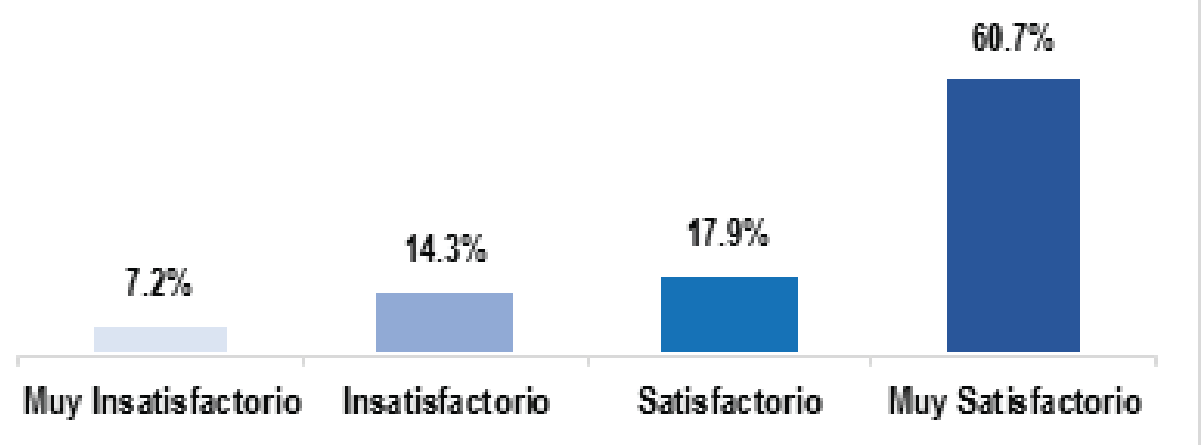

Fuente: Elaboración propia

\section{DISCUSIÓN}

En base a los resultados obtenidos en los gráficos 1 y 2 acerca de la actitud positiva de los estudiantes hacia los conocimientos de las ciencias químicas en los grupos control y experimental tanto en el pre test como en el post test. Se demuestra que ambos grupos presentan un porcentaje de $45.76 \%$ de actitudes positivas hacia el 
estudio de las ciencias, siendo este un factor necesario para evitar la falta de interés y poca fijación de conocimiento del estudiante (Puga García, 2017). El gráfico 2 demuestra que luego de la aplicación de las estrategias didácticas constructivistas y tradicional en los grupos experimental y control respectivamente, ambos conservan el mayor porcentaje en la actitud positiva hacia el estudio de la ciencia en la categoría de muy favorable. A pesar de la diferencia insignificante de apenas el $2.9 \%$, que podría indicar que una pequeña parte de los estudiantes demostró menos actitud positiva hacia el estudio de la química que el resto de los demás.

El análisis del conocimiento sobre el tema en ciencia que se desea desarrollar mediante la comparación de la media de respuestas correctas indica un mayor valor en el grupo experimental que en el grupo al cual se le aplicó estrategias didácticas tradicionales, tal como lo indica la Tabla 1. Sin embargo esta diferencia no es significativa, ya que el valor de significancia bilateral es de apenas 0.74 , un valor mayor al parámetro de comparación obtenido con un $95 \%$ de confianza correspondiente al coeficiente de correlación significativo al nivel 0.05 , con un valor de -0.647 indicados en la Tabla 2 y 3 . Por ello que se acepta la hipótesis nula, es decir que independientemente de la estrategia didáctica que se aplique, el conocimiento previo resultó igual en ambos grupos. En cambio según la tabla 4, la evaluación de los grupos después de la aplicación de sus respectivas estrategias didácticas indican que la media de respuestas correctas fue mayor en el grupo experimental al cual se le aplicaron estrategias didácticas constructivistas, a pesar que la significancia bilateral se mantiene en un valor insignificantemente mayor de 0.072 , en relación al parámetro de comparación obtenido con un $95 \%$ de confianza correspondiente al coeficiente de correlación significativo al nivel de 0.05 , con un valor de -0.211 , indicados en las tablas 5 y 6. Logrando identificar una diferencia apreciable en el grupo al que se le aplicó estrategias constructivistas, ya que presenta una mayor fijación de conocimientos en relación al grupo que fue tratado con la forma tradicional.

En el análisis de logro de competencias básicas (comunicación, aprendizaje colaborativo, resolución de problemas, análisis crítico reflexivo en el área de ciencias químicas y creatividad) mediante la aplicación del pre-test y según los resultados expuestos en los gráficos 3 y 4 , se observa que los valores más altos en el grupo tradicional se encuentran en satisfactorio y muy satisfactorio con un valor en ambos criterios de $37.5 \%$, esto indica que los estudiantes de química general poseen ciertas competencias previas adquiridas en el trascurso de su formación preuniversitaria al igual que el grupo experimental, el cual presenta porcentajes de $28.8 \%$ y $47.5 \%$ en las opciones de muy satisfactorio y satisfactorio respectivamente. 
Con respecto a los resultados obtenidos en el post test luego de la aplicación de estrategias didácticas correspondientes en cada grupo, se puede apreciar que no hubo un aumento significativo en el desarrollo de competencias en el grupo con estrategias didácticas tradicionales ya que mantuvo sus porcentajes iniciales y finales casi iguales. En cambio en el grupo experimental se observó que el porcentaje de estudiantes que pertenecía a la escala de muy satisfactorio en el pre-test con un valor $47.46 \%$ aumentó a un valor de $60.71 \%$, es decir que estos estudiantes potenciaron el desarrollo de sus competencias básicas con una diferencia significativa de $13.25 \%$. Tal como se muestran en los gráficos 5 y 6 .

\section{CONCLUSIONES}

En los grupos control y experimental se presenta en general una actitud positiva muy favorable hacia el estudio de las ciencias revelado en el pre-test, y luego de la aplicación de las estrategias didácticas correspondiente a cada grupo se mantiene el rango de favorable y muy favorable evidenciado en el post-test, lo que indica que las estrategias didácticas de ambos grupos provocan un aumento de actitudes positivas hacia el estudio de las ciencias, disminuyendo las actitudes negativas.

Ambos grupos presentan un porcentaje muy satisfactorio de competencias básicas antes de la aplicación de estrategias didácticas correspondientes a cada grupo, posteriormente a su aplicación el grupo experimental al que se le aplicó estrategias didácticas constructivista presentóó un aumento considerable en la adquisición y desarrollo de competencias, esto que se evidencia en el análisis confirmatorio.

\section{AGRADECIMIENTOS}

Al Doctor Olvis Castro por su respaldo académico en el área pedagógica al validar los instrumentos de medición.

Al joven estudiante de ingeniería Josué Ovidio Hernández Rivera por el apoyo en el análisis Estadístico de la información, donde también es valioso su aporte técnico. 


\section{REFERENCIAS BIBLIOGRÁFICAS}

Angulo, L. M. (2005). Programa para la Mejora de la Docencia Universitaria (1a ed.). Madrid, España: Pearson-Prentice Hall.

Delors, J. (1994). Los cuatro pilares de la educacion, la educacion encierra un tesooro, pp 91-103, recopilado de www. uv.mx/dgdaie/files/2012/11/cpp-d-c-Delors-los4pilaresdelaeducacion.pdf

Díaz Barriga Arcejo, F., Hernandez G. (2010). Estrategias Docentes, una interpretación constructivista ( $3^{\circ}$ ed.). D.F., México: McGraw-Hill.

Ortiz Uribe, F.G. , Garcia, M. (2010). Metodología de la Investigación, el Proceso y sus Técnicas (1a ed.). D.F., México: Limusa S.A. de C.V.

González , J., \& Wagenaar, R. (2003). TUNING EDUCATIONAL STRUCTURES IN EUROPE. Bilbao: University of Deusto.

Maldonado, A. (2001). Aprendizaje y Comunicación ¿Cómo Aprendemos? D.F., México: Pearson Educación.

Mayer, R. (2004). Psicología de la Educación (1a ed., Vol. II). Madrid, España: PearsonPrentice Hall.

Monereo, C., \& Pozo, J. I. (2003). La universidad ante la nueva cultura educativa: enseñar y aprender para la autonomía. Sintesis.

Montenegro, I. A. (2003). Aprendizaje y Desarrollo de las Competencias (1a ed.). Bogotá, Colombia.

Puga Garcia, A. (2017). Carrera de medicina: Estudiantes desmotivados para estudiar o .profesores pocos motivados para enseñar. Gaceta medica Esperituana, 19(2).

Sampieri, R. H. (2014). Metodología de la Investigación (6º ed.). D.F., México: McGraw-Hill. Torres Colin, R., Duno de Stefano, R., \& Can, L. L. (2009). El género Bauhinia (Fabaceae, Caesalpinioideae, Cercideae) en la península de Yucatan, Belice y Guatemala. Revista Mexicana de Biodiversidad, 293-301. 